\title{
Supercapacitor Leakage in Energy-Harvesting Sensor Nodes: Fact or Fiction?
}

\author{
Geoff V. Merrett, Alex S. Weddell \\ Electronics and Computer Science \\ University of Southampton, UK, SO17 1BJ \\ \{gvm, asw\}@ecs.soton.ac.uk
}

\begin{abstract}
As interest in energy-harvesting sensor nodes continues to grow, the use of supercapacitors as energy stores or buffers is gaining popularity. The reasons for their use are numerous, and include their high power density, simple interfacing requirements, simpler measurement of state-ofcharge, and a greater number of charging cycles than secondary batteries. However, supercapacitor energy densities are orders of magnitude lower. Furthermore, they have been reported to exhibit significant leakage, and this has been shown to increase exponentially with terminal voltage (and hence stored energy). This observation has resulted in a number of algorithms, designs and methods being proposed for effective operation of supercapacitor-based energy-harvesting sensor nodes. In this paper, it is argued that traditional 'leakage' is not as significant as has commonly been suggested. Instead, what is observed as leakage is in fact predominantly due to internal charge redistribution. As a result, it is suggested that different approaches are required in order to effectively utilize supercapacitors in energy-harvesting sensor nodes.
\end{abstract}

\section{INTRODUCTION}

Wireless sensor networks (WSNs) have been receiving growing attention for over a decade, and are beginning the transition from the research lab into industry. Their integration with energy harvesting, where energy is extracted from the local environment to power the sensor node, has recently seen increasing interest [1]. Whilst batteries, which were traditionally the power sources for WSNs, are energyconstrained (in that they provide a 'reservoir' of energy to be consumed as required), energy harvesting devices are powerconstrained (that is, over time, they provide endless energy but finite power - usually in the order of tens of $\mu \mathrm{W}$ to a few $\mathrm{mW}$ ) [2]. As a result of this distinction, nodes utilizing energy harvesting do not need to store energy for prolonged periods; they need only to buffer enough to 'smooth' any variation in the availability of environmental energy.

Two options for buffering this energy are secondary (rechargeable) batteries, or supercapacitors (also known as ultracapacitors or electric double-layer capacitors). A major benefit of supercapacitors is that they have high power densities, allowing them to supply large currents efficiently; conversely, batteries suffer from the 'rate-discharge effect', where their efficiency is directly linked to the magnitude of the current being drawn [3]. This is particularly relevant to sensor nodes, as they typically consume energy in bursts of high current when the node is active. Further benefits of supercapacitors are typically considered to be simpler charging requirements, easier measurement of the state-of-charge, and less degradation with cycled recharging (whereas secondary batteries see a reduction in the available capacity with each charge cycle) [5]. While supercapacitors do feature energy densities that are orders of magnitude lower than batteries (that is, for a given volume, the amount of energy stored in a battery will be much higher than a supercapacitor), energy harvesting sensor nodes do not need to be able to store large amounts of energy. As a result, supercapacitors have commonly been used as energy stores in energy harvesting sensor nodes [4]-[6].

One major disadvantage of supercapacitors that is commonly reported is the high leakage (also known as selfdischarge) that they exhibit. Leakage is often considered to be exponentially related to the terminal voltage or energy stored in the supercapacitor; indeed we made this same observation (shown in Fig. 1) during our early work in this area [7]. This leakage behavior is not unknown to the WSN community, and it has been reported in many publications [4], [8], [9]. Furthermore, a number of techniques have been reported in the literature which aim to adapt the behavior of the sensor node in order to accommodate this leakage property, for example by avoiding operating the supercapacitor in the regions where higher leakage is exhibited [10]. It is recognized that variation in leakage exists between devices of different capacities (as shown by our empirical results presented in Fig 2), but also of different manufacturers and even batches of the same device.

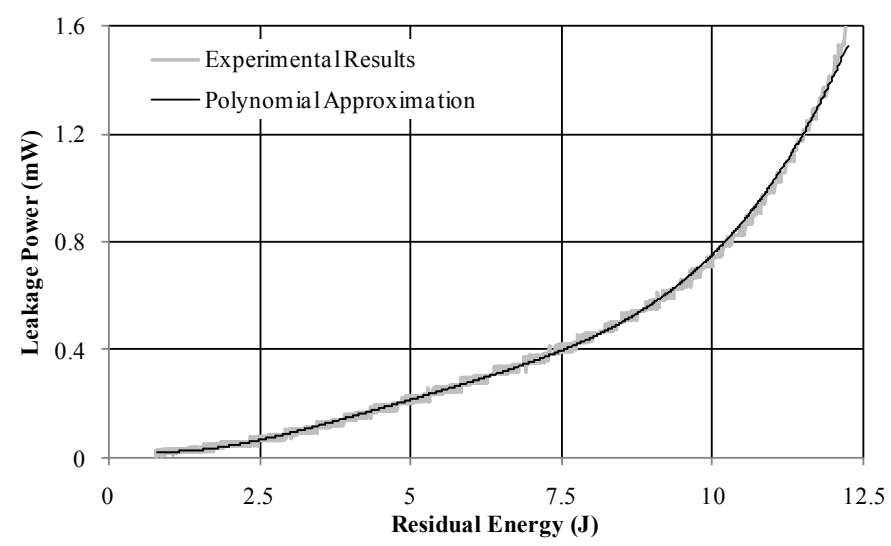

Figure 1. Estimated leakage power empirically measured from a $4.7 \mathrm{~F}$ supercapacitor (reproduced from our early work in this area [7]), highlighting how supercapacitor leakage apparently varies with stored energy (and therefore terminal voltage)
This work was supported in part by the Engineering and Physical Sciences Research Council (EPSRC) UK under grant EP/G067740/1 "Next Generation Energy-Harvesting Electronics: Holistic Approach," www.holistic.ecs.soton.ac.uk 


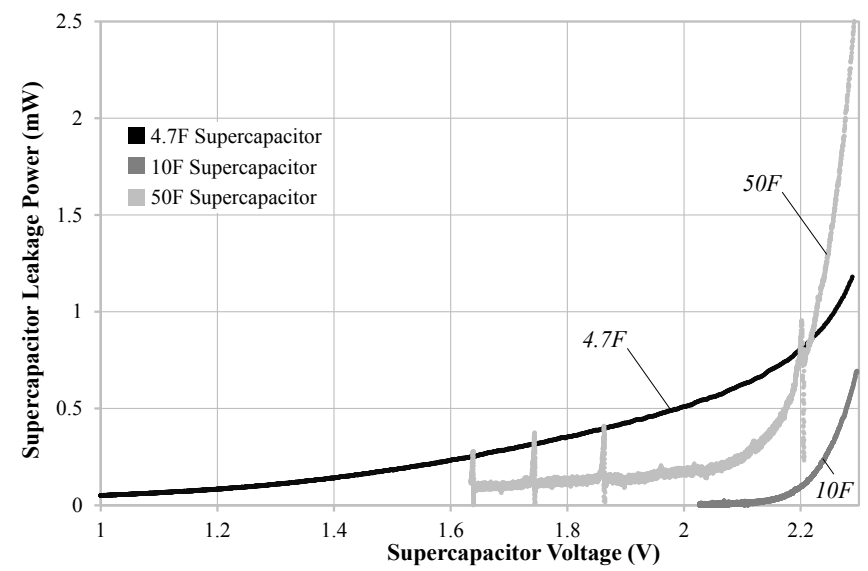

Figure 2. Empirical results obtained which highlights how supercapacitor leakage varies with rated capacitance.

Upon more detailed investigation and experimentation, anomalies begin to arise with this exponential model of the relationship between leakage power and terminal voltage. One example is when charging a supercapacitor to different voltages, or for different periods of time. To illustrate this, a fully discharged Panasonic Gold [11] 4.7F supercapacitor was connected to a power supply at either $1.8 \mathrm{~V}$ or $2.3 \mathrm{~V}$, and left for $1 \mathrm{hr}, 10 \mathrm{hrs}$ or $100 \mathrm{hrs}$. After the charge period elapsed, the leakage was monitored, and the results plotted in Fig. 3.

As the results in Fig 3 show, different combinations of these charging parameters cause dramatically different leakage behavior. The reasons for this are explored in the remainder of this paper.

\section{LEAKAGE AND CHARGE REDISTRIBUTION}

In this section, the behavior of supercapacitor leakage is explored further through empirical results and simulation. It is proposed that actual leakage is not as significant as has commonly been suggested. Instead, what is observed as leakage is in fact predominantly due to internal charge redistribution within the supercapacitor.

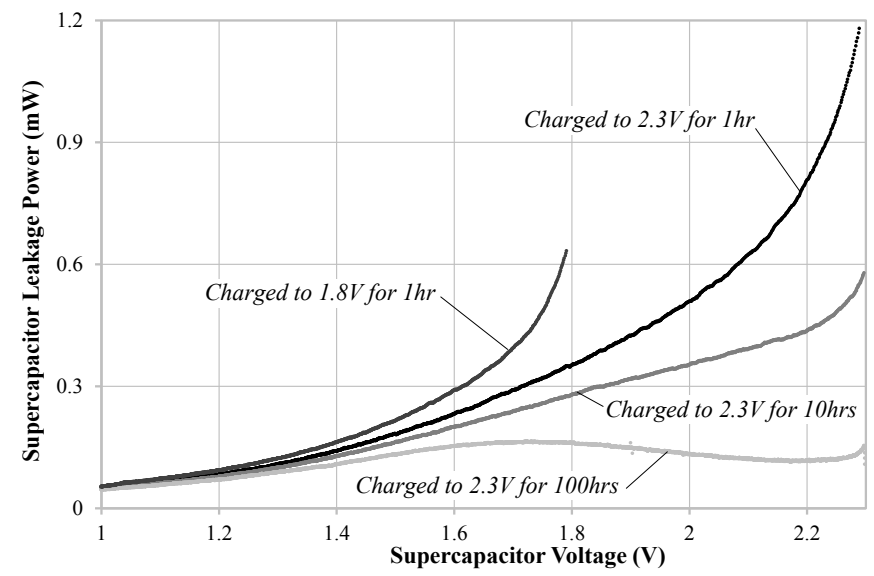

Figure 3. Empirical results highlighting how the leakage from a $4.7 \mathrm{~F}$ supercapacitor varies with the period of time for which it was charged (and the voltage it was charged to).

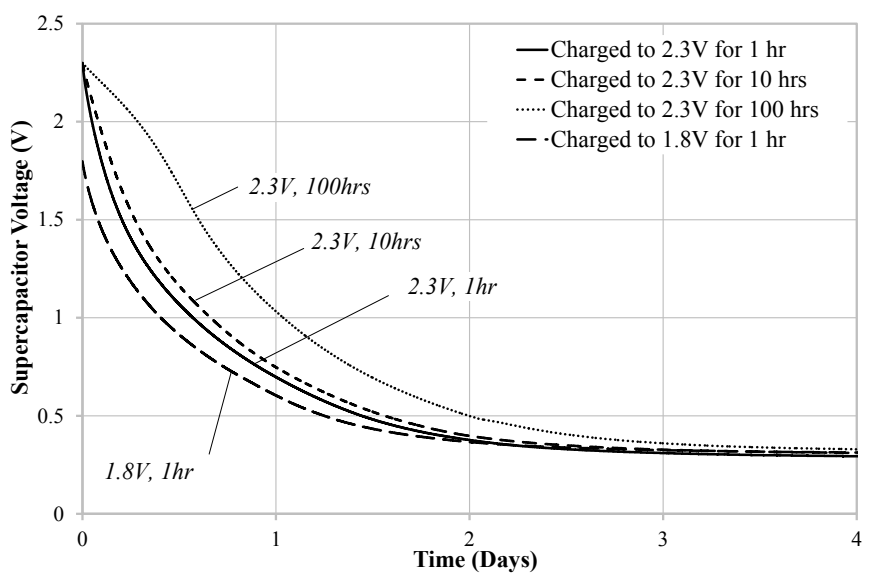

Figure 4. Empirical results highlighting how the terminal voltage on a $4.7 \mathrm{~F}$ supercapacitor drops during self-discharge, and how this varies with the period of time for which it was charged (and the voltage it was charged to).

\section{A. Method for Obtaining Empirical Leakage Results}

To understand the cause of the differing leakage behavior observed in Fig 3, the process used to arrive at this plot needs to be fully understood. Having charged the supercapacitor to a particular voltage for a predefined period of time, it was left disconnected while the drop in terminal voltage was observed and recorded. This measured data is plotted in Fig 4.

This dataset of voltage-time pairs were subsequently processed to calculate the energy in the supercapacitor, and then differentiated to give instantaneous leakage power. These processed data are shown in Fig 3. The energy in the supercapacitor was calculated using the equation for energy in an ideal capacitor (1).

$$
E=\frac{1}{2} C V^{2}
$$

In practice, a supercapacitor is a non-ideal device, and (1) does not reflect the actual relationship between the terminal voltage and stored energy. Equivalent circuit models for supercapacitors have been proposed for decades, though these have generally been aimed at 'large' supercapacitors with capacitances of many hundreds of Farads (such as those proposed for use in electric vehicles) [12]. These models all share a representation of a supercapacitor having a number of time constants which cover many orders of magnitude (in fact, most of the different equivalent models proposed can be transformed to one-another from a generic model [13]). One of these, the 'ladder-model', is shown in Fig. 5. In this equivalent circuit, each of the three branches of the ladder have differing time constants, and resistor Rleak represents the true 'leakage' element. For simplicity, the voltage-dependent capacitance $\mathrm{Cv}$ will be neglected for the remainder of this paper.

Zubietta et al. [14] proposed a method for characterizing a particular supercapacitor to identify the component values that are present in the model of Fig 5; this was subsequently extended by Weddell et al. [15] with application to 'smaller' supercapacitors such as those used in WSNs. 


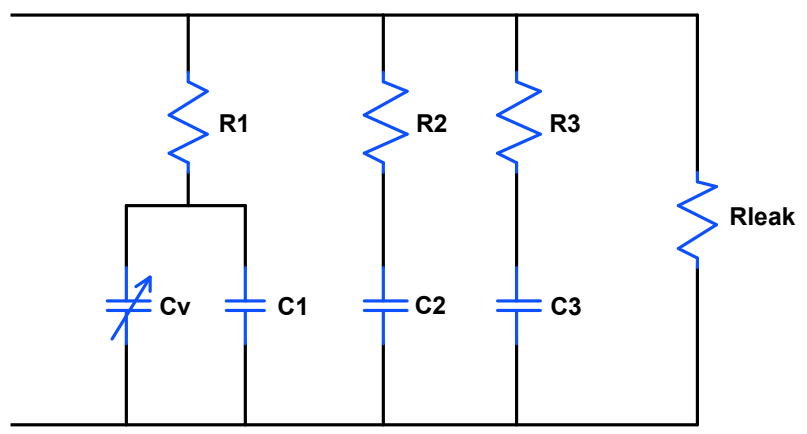

Figure 5. Equivalent circuit of a supercapacitor [14].

\section{B. Simulating Supercapacitor Leakage Behavior}

To further investigate this leakage behavior, simulations were performed in LTSpice of the equivalent circuit shown in Fig. 5. The component values used in the simulations are shown in Table I, and are intended to illustrate behavior rather than represent a particular characterized device. A transient analysis was performed on three simulated supercapacitors which were charged to $2.3 \mathrm{~V}$ for $0.1,1$ and 10 hours. The voltages across and currents through all components in the equivalent models were logged for further analysis.

Fig. 6a shows how the supercapacitors' terminal voltage drops after the simulated power supply is disconnected at $t=0$. As expected from the empirical results in Fig. 4, the longer the supercapacitor has been charged for, the slower its voltage drops. If we plot the total energy stored inside the supercapacitor, i.e. the sum of energies stored in C1, C2 and $\mathrm{C} 3$, calculated using (1), it can be observed that there is more energy stored in those that have been charged for longer (shown in Fig. 6). In the simulated example, the supercapacitor charged for $10 \mathrm{hrs}$ contains twice as much energy at $t=0$ than the one charged for $0.1 \mathrm{hrs}$. This effect is due to the different time constants present in the different branches of the ladder in the equivalent circuit. When charging the supercapacitor, the terminal voltage rises rapidly as the first branch (with the shortest time constant) charges quickly, whereas the other branches take a lot longer.

In the simulated supercapacitor, branch three has a time constant of over 11 hours. Once charging stops, the slower branches continue to charge, taking their energy from the faster branches. This redistribution of charge causes a drop in the terminal voltage, which is commonly perceived as leakage. This behavior can be seen in Fig. 7, which plots the energy in each branch of the simulated supercapacitor charged for $1 \mathrm{hr}$. It can be seen that the first branch charges almost instantly, whereas the second and third branches do not get fully charged before $t=0$. Once the power supply is disconnected, the first branch begins to discharge into the second and third, thus causing a drop in the observed terminal voltage.

TABLE I. PARAMETER VALUES USED IN SIMULATIONS

\begin{tabular}{|r|l||r|l|}
\hline Parameter & Value & Parameter & Value \\
\hline $\mathrm{C} 1$ & $2.5 \mathrm{~F}$ & $\mathrm{R} 1$ & $1 \Omega$ \\
$\mathrm{C} 2$ & $1.5 \mathrm{~F}$ & $\mathrm{R} 2$ & $1 \mathrm{k} \Omega$ \\
$\mathrm{C} 3$ & $4 \mathrm{~F}$ & $\mathrm{R} 3$ & $10 \mathrm{k} \Omega$ \\
$\mathrm{Cv}$ & $0 \mathrm{~F}$ & Rleak & $20 \mathrm{k} \Omega$ \\
\hline
\end{tabular}
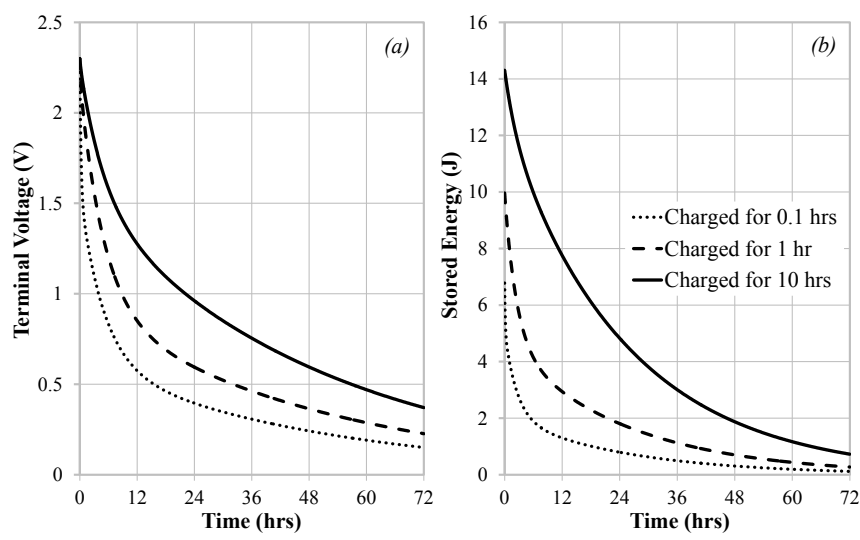

Figure 6. Simulation results showing how a) the terminal voltage and b) the stored energy are affected by varying the period of time for which the supercapacitor is charged.

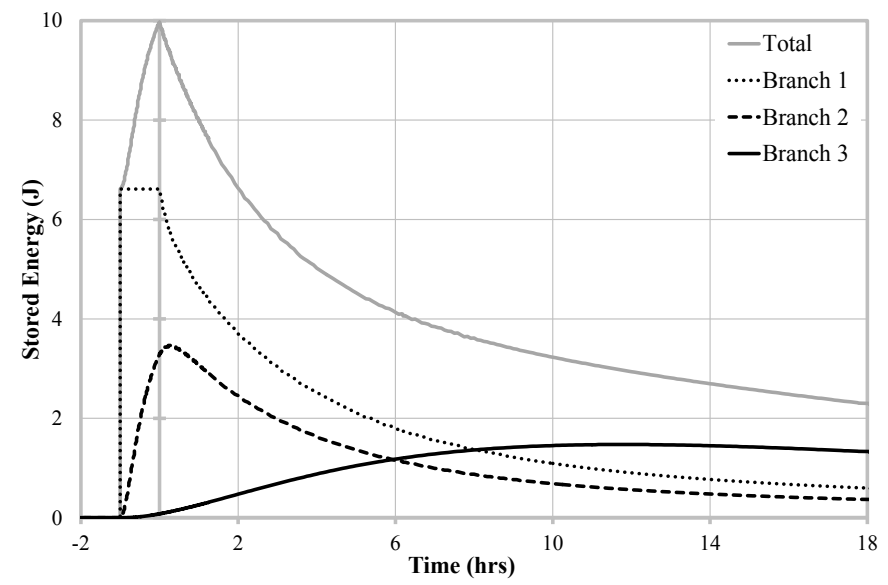

Figure 7. Simulation results showing the energy in each branch of the supercapacitor (charged for $1 \mathrm{hr}$ ), and the total energy stored. The power supply was disconnected at $t=0$.

This 'redistribution' of charge within the supercapacitor could, intuitively, lead to the understanding that leakage is not as considerable a problem as initially thought. If charge is simply being redistributed around the supercapacitor, is this drop in voltage an insignificant artifact (where in fact very little energy is being lost)? However, this is clearly not the case as Figs. 6 and 7 show that the total energy in the supercapacitor is dropping at a reasonable rate. This energy loss is due to the redistribution process itself; in the equivalent circuit of Fig. 5, consider the power dissipated in each of the branch resistances while charge is being transferred from one branch to another.

As a result of this analysis, in this paper we refer to the energy losses as having two components: those caused by leakage (i.e. energy dissipated by resistor Rleak) and those caused by charge redistribution (i.e. energy dissipated by resistors $\mathrm{R} 1, \mathrm{R} 2$ and $\mathrm{R} 3$ ).

By further analyzing the simulation results by applying the equation for power dissipated by a resistor $(P=I V)$, the powers dissipated due to leakage and charge redistribution can be calculated. These results are shown, and plotted against the 


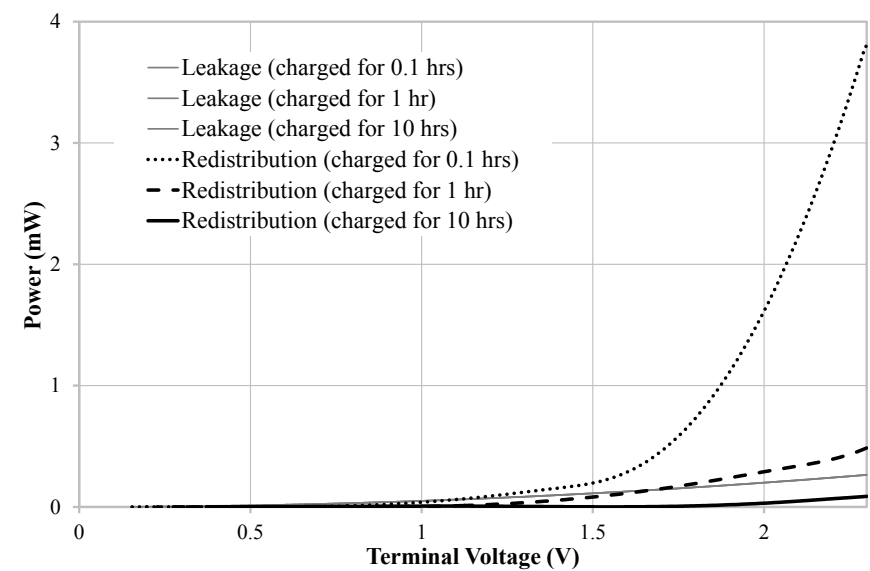

Figure 8. Simulation results showing the leakage and redistribution powers present at different voltages for various periods of time for which the supercapacitor is charged.

terminal voltage, in Fig. 8. This highlights that the true 'leakage' is actually reasonably small, with a maximum of just over $250 \mathrm{nW}$ (as expected, due to the leakage being modeled in Fig. 5 as a purely resistive load). As a result, for the supercapacitor charged for only $0.1 \mathrm{hrs}$, the predominant loss of energy is due to the redistribution of charge between branches. The longer that the supercapacitor is charged for, the lower this redistribution power becomes.

The results discussed above and presented in Fig. 8 may give the impression that energy loss is low if a supercapacitor has been charged for an extended period; a supercapacitor that has been charged for a longer period could be considered to lose less energy through redistribution and therefore lose less energy overall. However, through inspection of Fig. 9 (which compares the energy lost through leakage and redistribution for a supercapacitor charged for 0.1 and $10 \mathrm{hrs}$ ) we see that, in fact, the supercapacitor charged for longer loses twice the energy in the same time period. It can also be observed that, while the energy lost through leakage and redistribution are similar in the case of the supercapacitor charged for $0.1 \mathrm{hrs}$, leakage is almost an order of magnitude greater in the supercapacitor charged for $10 \mathrm{hrs}$.

This behavior is due to the fact that the supercapacitor charged for 0.1 hrs very rapidly drops its voltage, reducing the energy lost through leakage (it can be seen from Fig. 8 that leakage overtakes redistribution when the voltage drops below $\sim 1 \mathrm{~V})$. In the case of the supercapacitor charged for $10 \mathrm{hrs}$, the voltage remains high for a longer period of time and, due to the little redistribution that takes place, the power consumed through redistribution is always lower than leakage (Fig. 8).

\section{DISCUSSION}

The impact of this analysis may, at first, be unclear. Inspecting Fig. 6b, it appears that both supercapacitors are eventually depleted at not too dissimilar times. However, two key observations should be made. Firstly, the voltage drops considerably faster with shorter charge times. WSN power circuitry can usually only operate down to a lower voltage threshold (typically in the range of $1-2 \mathrm{~V}$ ), and hence the longer
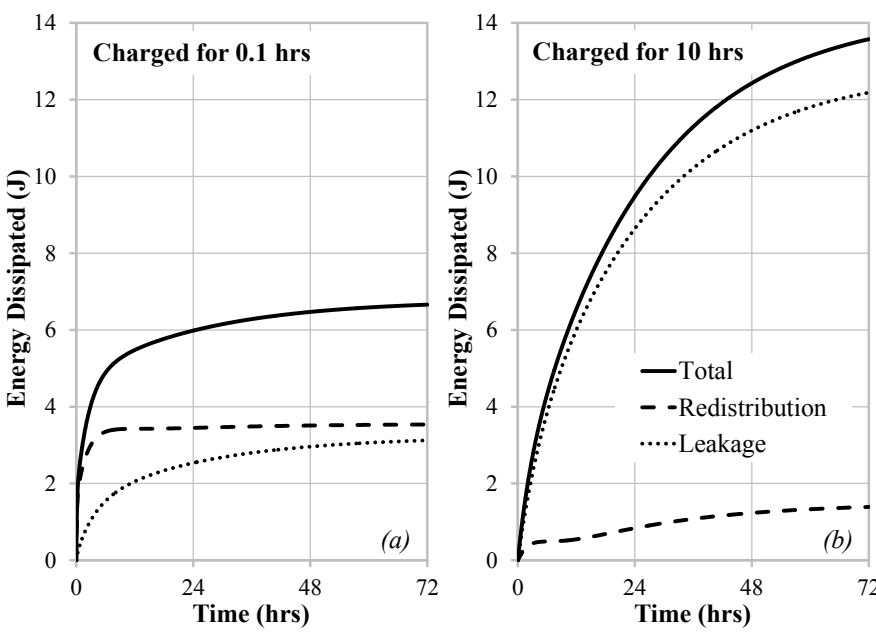

Figure 9. Simulation results showing the proportion of energy lost through redistribution and leakage, after being charged for a) 0.1 hours, or b) 10 hours.

charge times can provide a usable voltage for considerably longer. Secondly, this effect is accentuated when the supercapacitor is also loaded. Consider, for example, a sensor node powered by the supercapacitor when no energy is being harvested (for example, the sun has just set). This scenario was simulated with the three supercapacitor scenarios shown above, each consuming a constant current roughly equivalent to a typical sensor node duty cycled at $0.1 \%$; the results are presented in Fig. 10. From these results, it can be observed that the node is able to operate for considerably longer when charged for a longer period.

Much of the existing leakage-aware research has assumed that all of the energy losses experienced are due to leakage. From the results shown in Fig. 10, it could be considered that a sensor node that has been charging for $>10 \mathrm{hrs}$ (for example, harvesting all day from a solar cell) will be able to operate for a prolonged period overnight. Conversely, an algorithm which has performed many shorter discharge-charge cycles throughout the day, would not be able to operate for as long overnight.

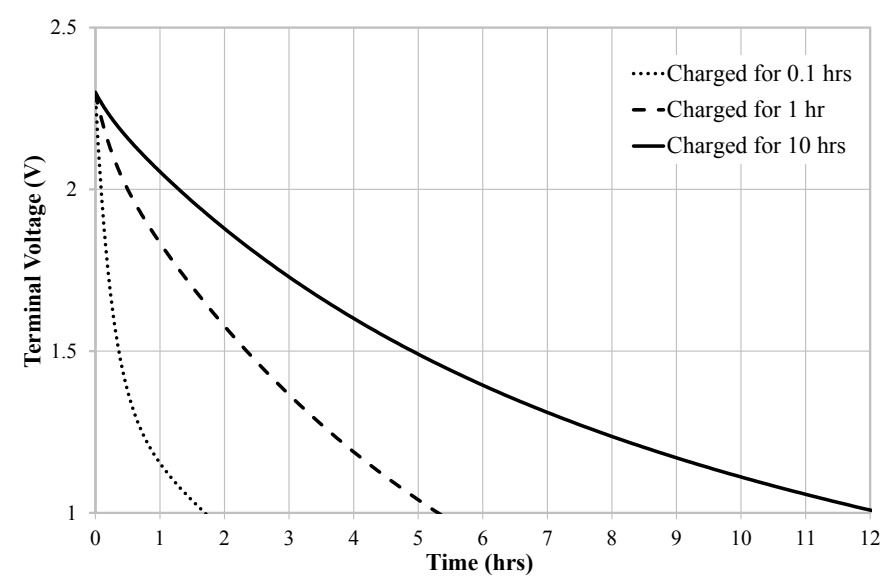

Figure 10. Simulation results showing the terminal voltage of the supercapacitor previously simulated, but this time loaded with a constant current load of 30nA (approximately equivilent to a 30mA sensor node duty cycled at $0.1 \%$ ). 
A further observation can be made with respect to the commonly quoted advantages of supercapacitors, listed in the introduction to this paper. First, it appears that an effect similar to battery relaxation is present in a supercapacitor, in the form of the charge redistribution discussed. Furthermore, the perceived ease of state-of-charge measurement is in fact naïve, as the supercapacitor's terminal voltage cannot be reliably translated into stored energy through the ideal capacitor equation (1). This is not to say that supercapacitors are not suitable choices for energy stores in energy harvesting sensor nodes; indeed they offer many advantages to these applications. Instead, it is observed that these properties need to be understood in designing efficient supercapacitor sensor nodes.

While the relationship between the charge time and leakage behavior explored in this paper has been previously recognized (for example in manufacturers' documentation [11]), this paper has investigated the relative magnitudes and behaviors of these two components. It is therefore hoped that this paper will highlight these effects to the WSN community, and encourage researchers to think about the impact of this rather more complex behavior when designing sensor nodes.

\section{REFERENCES}

[1] S. Beeby and N. White, Eds., Energy Harvesting for Autonomous Systems, Norwood, MA: Artech House, 2010.

[2] S. Roundy, P. K. Wright and J. M. Rabaey, Energy Scavenging for Wireless Sensor Networks, Norwell, MA: Kluwer Academic, 2003.

[3] C. Park et al., "Battery discharge characteristics of wireless sensor nodes: an experimental analysis," in IEEE Conf. Sensor \& Ad-Hoc Communications and Networks (SECON), 2005, pp. 430-440.
[4] X. Jiang et al., "Perpetual environmentally powered sensor networks," in Int'l Symp. Information Processing in Sensor Networks (IPSN), 2005, pp. $463-468$.

[5] F. Simjee and P. H. Chou, "Everlast: long-life, supercapacitor-operated wireless sensor node," in Int'l Symp. Low Power Electronics and Design (ISLPED), 2006, pp. 197-202.

[6] R. N. Torah et al., "Self-powered autonomous wireless sensor node using vibration energy harvesting," Measurement Science and Technology, vol. 19, no. 12, 2008.

[7] G. V. Merrett et al., "An empirical energy model for supercapacitor powered wireless sensor nodes," in Int'l Conf. Computer Communications and Networks (ICCCN), 2008.

[8] T. Zhu et al., "eShare: a capacitor-driven energy storage and sharing network for long-term operation," in ACM Conf. Embedded Networked Sensor Systems (SenSys ), 2010, pp. 239-252.

[9] C. Renner, J. Jessen and V. Turau, "Lifetime prediction for supercapacitor-powered wireless sensor nodes," in Proc. GI/ITG KuVS Fachgespräch 'Drahtlose Sensornetze' (FGSN), 2009, pp. 55-58.

[10] T. Zhu et al., "Energy-synchronized computing for sustainable sensor networks," Ad Hoc Networks, to be published (available online 2010).

[11] Panasonic Electronic Devices Co. Ltd., "Technical guide of electric double layer capacitors - edition 7.4," 2011, last accessed April 2012, http://industrial.panasonic.com/www-ctlg/tech/tABC0000_AM.html.

[12] S. Lisheng and M. L. Crow, "Comparison of ultracapacitor electric circuit models," in IEEE Power \& Energy Society General Meeting Conversion and Delivery of Electrical Energy in the 21st Century, 2008.

[13] R. Faranda, M. Gallina, and D. T. Son, "A new simplified model of double-layer capacitors," in Int'l Conf. Clean Electrical Power (ICCEP), 2007, pp. 706-710.

[14] L. Zubieta and R. Bonert, "Characterization of double-layer capacitors for power electronics applications," IEEE Trans. Ind. Appl., vol. 36, no. 1, pp. 199-205, Jan/Feb 2000.

[15] A. S. Weddell et al., "Accurate supercapacitor modeling for energyharvesting wireless sensor nodes," IEEE Trans. Circuits Syst. II: Exp. Briefs, vol. 58, no. 12, pp. 911-915, Dec. 2011. 